\title{
ADUBAÇÃO VERDE NA RECUPERAÇÃO DA FERTILIDADE DE UM LATOSSOLO VERMELHO-ESCURO DEGRADADO1
}

\author{
FLÁVIAAPARECIDA DE ALCÂNTARA², ANTONIO EDUARDO FURTINI NETO3, \\ MIRALDA BUENO DE PAULA ${ }^{4}$, HUGO ADELANDE DE MESQUITA ${ }^{5}$ e JOEL AUGUSTO MUNIZ ${ }^{6}$
}

\begin{abstract}
RESUMO - O experimento foi conduzido na Fazenda Experimental Nova Baden, da Empresa de Pesquisa Agropecuária de Minas Gerais (EPAMIG), em Lambari, MG, objetivando avaliar o desempenho dos adubos verdes guandu (Cajanus cajan (L.) Millsp.) e crotalária-júncea (Crotalaria juncea L.), com e sem incorporação da biomassa, na recuperação da fertilidade de um solo (Latossolo Vermelho-Escuro distrófico) degradado, cultivado com braquiária (Brachiaria decumbens Stapf.). Empregou-se o delineamento experimental de blocos casualizados em esquema fatorial de parcelas subdivididas, com três repetições, colocando-se nas parcelas o manejo da biomassa, e nas subparcelas, as leguminosas e a braquiária. As três culturas foram manejadas na fase final de florescimento das leguminosas, quando se fizeram amostragens de biomassa para determinação de produção de matéria seca, concentração e conteúdo de nutrientes. Realizaram-se avaliações de amostras de solo em três épocas $(90,120$ e 150 dias após o manejo) e seis profundidades (0-5, 5-10, 10-20, 20-40, 40-60 e 60-80 cm). O guandu se destacou na primeira avaliação quanto às melhorias na fertilidade do solo, e a crotalária-júncea, de mineralização mais lenta, na segunda. Na terceira avaliação, não foram encontrados benefícios dos adubos verdes nas propriedades químicas do solo.
\end{abstract}

Termos para indexação: adubo verde, Cajanus cajan, Crotalaria juncea, manejo da biomassa.

\section{GREEN MANURING IN THE RECOVERY OF DEGRADED OXISOIL FERTILITY}

\begin{abstract}
The experiment was conducted at the Empresa de Pesquisa Agropecuária de Minas Gerais, Nova Baden Experimental Station, in Lambari, MG, Brazil, aiming at evaluating the performance of the green manures pigeonpea (Cajanus cajan (L.) Millsp.) and sunnhep (Crotalaria juncea L.) with and without incorporation of the biomass, on the recovery of fertility of degraded soil cultivated with brachiaria (Brachiaria decumbens Stapf.). Experimental design used was of randomized block in factorial scheme of split plots with three replications, putting the biomass handling in the plots and both the legumes and brachiaria in the subplots. The three crops were cut and handled at the late flowering stage of the legumes, when samplings of the plant material were done for assessing dry matter yield, concentration and amount of nutrient. Evaluations of soil samplings at six depths (0-5, 5-10, $10-20,20-40,40-60$ e $60-80 \mathrm{~cm}$ ) and three periods (90, 120 and 150 days after handling) were performed. Pigeonpea stood out in the first evaluation concerning the improvements in soil fertility and sunnhep and slower mineralization, in the second. In the third evaluation, no benefits from the green manure on the chemical properties of the soil were found.
\end{abstract}

Index terms: green manure, Cajanus cajan, Crotalaria juncea, biomass handling.

\footnotetext{
${ }^{1}$ Aceito para publicação em 15 de março de 1999.

${ }^{2}$ Eng. Agrôn., M.Sc., aluna do CPGSNP, DCS, UFLA, Caixa Postal 37, CEP 37200-000 Lavras, MG. Bolsista da CAPES. E-mail: falcanta@ufla.br

${ }^{3}$ Eng. Agrôn., Dr., DCS, UFLA, Lavras, MG. Bolsista do CNPq. E-mail: afurtini@ufla.br

${ }^{4}$ Eng. Agrôn., Dr., EPAMIG, Caixa Postal 176, CEP 37200-000 Lavras, MG. E-mail: epamig@ufla.br

${ }^{5}$ Eng. Agrôn., M.Sc., EPAMIG. E-mail: epamig@ufla.br

${ }^{6}$ Eng. Agrôn., Dr., DEX, UFLA. E-mail: joamuniz@ufla.br
}

\section{INTRODUÇÃO}

Atualmente, a preocupação com o avanço do processo degradativo instalado em grande parte dos solos brasileiros, e com a prevenção da degradação de novas áreas, tem conduzido à necessidade do uso de práticas de adição de matéria orgânica ao solo. Entre essas, destaca-se a adubação verde, reconhecida como uma alternativa viável na busca da sustentabilidade dos solos agrícolas. Segundo 
Andrade et al. (1984), para a recuperação de pastagens degradadas essa prática se destaca em importância e viabilidade, pois a utilização de leguminosas conduz ao aumento ou à manutenção do teor de $\mathrm{N}$ do solo, diretamente correlacionado com a presença da matéria orgânica.

A família das leguminosas é a mais utilizada como adubo verde. De acordo com Miyasaka et al. (1984), a principal razão para essa preferência está em sua capacidade de fixar o $\mathrm{N}$ atmosférico mediante a simbiose com bactérias do gênero Rhyzobium/Bradyrhyzobium nas raízes. Outros motivos citados pelos autores são seu alto teor de compostos orgânicos nitrogenados e a presença de um sistema radicular geralmente bem profundo e ramificado, capaz de extrair nutrientes das camadas mais profundas do solo.

A produção de massa vegetal do adubo verde é um aspecto de grande importância, pois algumas espécies apresentam um grande crescimento vegetativo. Alvarenga (1993), comparando diferentes adubos verdes, concluiu ser o guandu a espécie de maior potencial para penetração de raízes no solo, maior produção de massa seca e maior quantidade de nutrientes imobilizados nas condições por ele estudadas. Kiehl (1960), em trabalho com guandu, feijão-de-porco, mucuna-preta, mucuna-rajada, crotalária-júncea e crotalária-paulina, verificou que guandu e crotalária-júncea foram as espécies que mais se destacaram na produção de massa verde e matéria seca.

Entre os efeitos da adubação verde sobre a fertilidade do solo estão o aumento do teor de matéria orgânica; a maior disponibilidade de nutrientes; a maior capacidade de troca de cátions efetiva $(\mathrm{t})$ do solo; o favorecimento da produção de ácidos orgânicos, de fundamental importância para a solubilização de minerais; a diminuição dos teores de Al trocável através de sua complexação, e o incremento da capacidade de reciclagem e mobilização de nutrientes lixiviados ou pouco solúveis que estejam nas camadas mais profundas do perfil (Calegari et al., 1993).

Por outro lado, existem também evidências de falta de resposta dos adubos verdes à melhoria da fertilidade do solo. Camargo et al. (1968), estudando a influência da adubação verde com Crotalaria spectabilis Roth. e Secale sereale L. nas características químicas de uma Terra Roxa e de um Latossolo Vermelho-Amarelo, verificaram, após cinco meses de realização da prática, que não houve efeito significativo da adubação verde nos teores de $\mathrm{P}, \mathrm{K}$ e Ca desses solos. Também Caceres (1994) não constatou alterações expressivas no teor de nutrientes e matéria orgânica, no $\mathrm{pH}$, na capacidade de troca de cátions (CTC), soma de bases (SB) e saturação por Al do solo, após a utilização de sete diferentes espécies de adubos verdes, inclusive de crotalária-júncea e guandu, durante cinco meses.

Os efeitos promovidos pela adubação verde nas propriedades químicas do solo são bastante variáveis, dependendo de fatores como: a espécie utilizada, o manejo dado à biomassa, a época de plantio e corte do adubo verde, o tempo de permanência dos resíduos no solo, as condições locais, e a interação entre esses fatores.

Este trabalho teve como objetivo avaliar o desempenho de duas leguminosas utilizadas como adubo verde, em diferentes formas de manejo, na recuperação da fertilidade de um solo degradado, cultivado por longo período com pastagem formada por Brachiaria decumbens Stapf.

\section{MATERIAL E MÉTODOS}

O experimento foi conduzido na Fazenda Experimental Nova Baden, da Empresa de Pesquisa Agropecuária de Minas Gerais - EPAMIG -, situada em Lambari, região sul de Minas Gerais. O solo é um Latossolo Vermelho-Escuro distrófico com as seguintes características químicas e granulométricas (camada de $0-20 \mathrm{~cm}$ ): $\mathrm{pH}$ em água 4,6; $1,0 \mathrm{mg} / \mathrm{dm}^{3}$ de $\mathrm{P} ; 23,0 \mathrm{mg} / \mathrm{dm}^{3}$ de $\mathrm{K} ; 2,0 \mathrm{mmol}_{\mathrm{c}} / \mathrm{dm}^{3}$ de $\mathrm{Ca} ; 1,0 \mathrm{mmol}_{\mathrm{c}} / \mathrm{dm}^{3}$ de $\mathrm{Mg} ; 9,0 \mathrm{mmol}_{\mathrm{c}} / \mathrm{dm}^{3}$ de $\mathrm{Al}$; $98,0 \mathrm{mmol}_{\mathrm{c}} / \mathrm{dm}^{3} \mathrm{de} \mathrm{H}+\mathrm{Al} ; 3,6 \mathrm{mmol}_{\mathrm{c}} / \mathrm{dm}^{3}$ de SB; $12,6 \mathrm{mmol}_{\mathrm{c}} / \mathrm{dm}^{3} \mathrm{de} \mathrm{t} ; 101,6 \mathrm{mmol}_{\mathrm{c}} / \mathrm{dm}^{3} \mathrm{de} \mathrm{T} ; 71,0 \% \mathrm{de} \mathrm{m}$; $3,5 \%$ de V; $33 \mathrm{~g} / \mathrm{kg}$ de M.O.; $1,6 \mathrm{~g} / \mathrm{kg}$ de N-total; $5,0 \mathrm{mg} / \mathrm{dm}^{3}$ de $\mathrm{N}_{-} \mathrm{NO}_{3}^{-} ; 8,0 \mathrm{mg} / \mathrm{dm}^{3}$ de $\mathrm{N}-\mathrm{NH}_{4}^{+}$; $0,7 \mathrm{mg} / \mathrm{dm}^{3}$ de $\mathrm{Zn} ; 2,9 \mathrm{mg} / \mathrm{dm}^{3} \mathrm{de} \mathrm{Cu} ; 168,3 \mathrm{mg} / \mathrm{dm}^{3} \mathrm{de} \mathrm{Fe}$; $3,8 \mathrm{mg} / \mathrm{dm}^{3}$ de $\mathrm{Mn} ; 340 \mathrm{~g} / \mathrm{kg}$ de areia; $160 \mathrm{~g} / \mathrm{kg}$ de silte e $500 \mathrm{~g} / \mathrm{kg}$ de argila. A área experimental foi formada com Brachiara decumbens Stapf. no ano de 1982, sem a realização de quaisquer práticas de caráter conservacionista ou de correção e adubação do solo.

Foram constituídos seis tratamentos por meio de três culturas (guandu, crotalária-júncea e pastagem de braquiária já existente na área e utilizada como testemunha) e duas 
formas de manejo da biomassa (cortada e incorporada ao solo e cortada, e não incorporada). Utilizou-se um esquema de parcelas subdivididas instalado num delineamento de blocos casualizados, com três repetições, sendo avaliadas nas parcelas as formas de manejo, e nas subparcelas, as culturas. Foram realizadas, em cada subparcela, amostragens de solo em seis profundidades $(0-5,5-10$, 10-20, 20-40, 40-60 e 60-80 cm), caracterizando-se um experimento em faixa.

Para a instalação do ensaio, demarcou-se a área experimental, localizando-se parcelas e subparcelas, inclusive as que permaneceriam com a pastagem de braquiária, e procedeu-se à distribuição de calcário na área total, com dosagem calculada conforme a recomendação da Comissão de Fertilizantes do Solo do Estado de Minas Gerais (1989); realizou-se uma aração e uma gradagem para incorporação do corretivo, e 32 dias após a calagem procedeu-se ao plantio do guandu (4/12/1995). Devido a diferença de ciclo das leguminosas, a crotalária-júncea foi plantada aproximadamente 60 dias após (5/2/1996), a fim de que, ambas pudessem ser cortadas e manejadas na mesma época, o que, além de viabilizar a condução do experimento no campo, permitiu que os resíduos de ambas estivessem sujeitos às mesmas condições ambientais durante a mineralização. As leguminosas foram plantadas no espaçamento de $0,5 \mathrm{~m}$ entre linhas, com uma densidade de 20 e 50 sementes por metro linear, de guandu e de crotalária-júncea, respectivamente. Suas sementes foram infectadas com inoculante específico fornecido pela Embrapa-Centro Nacional de Pesquisa de Agrobiologia. O corte dos adubos verdes foi realizado com o auxílio de roçadeira, quando ambos se encontravam na fase final de florescimento e início da formação das vagens (27/4/1996). Nessa ocasião, também foi realizado o corte da pastagem. Antes do manejo da biomassa das culturas, foi realizada a amostragem de material vegetal (parte aérea) para posterior determinação de produção de matéria seca e concentração de nutrientes. Foram retiradas amostras do material vegetal cortado de cada cultura $\left(2 \mathrm{~m}^{2}\right)$, e misturou-se o proveniente das duas parcelas (onde seria e onde não seria feita a incorporação), perfazendo um total de três amostras por bloco. Cada amostra continha $1 \mathrm{~kg}$ de material vegetal, sendo o restante devolvido às respectivas parcelas. Quanto ao manejo da biomassa das culturas, nas parcelas onde esta foi incorporada, a operação foi feita através de gradagem a $10 \mathrm{~cm}$ de profundidade.

Foram realizadas três avaliações da fertilidade do solo, amostrando-se aos 90, 120 e 150 dias após o manejo das culturas. Retirou-se uma amostra composta (cinco subamostras/composta) por subparcela para cada profundidade.
Nas plantas, avaliou-se a produção de matéria seca e suas concentrações de nutrientes, determinando-se o N, P, $\mathrm{K}, \mathrm{Ca}, \mathrm{Mg}, \mathrm{S}, \mathrm{B}, \mathrm{Cu}, \mathrm{Fe}, \mathrm{Mn}$ e $\mathrm{Zn}$ na parte aérea dos adubos verdes e da pastagem, segundo Malavolta et al. (1989). Os extratos da matéria seca de parte aérea foram obtidos por digestão nitroperclórica, exceto no $\mathrm{B}$, cuja extração foi feita por via seca. $\mathrm{P} \mathrm{e} \mathrm{B} \mathrm{foram} \mathrm{determinados} \mathrm{por}$ colorimetria; $\mathrm{Ca}, \mathrm{Mg}, \mathrm{Cu}, \mathrm{Fe}, \mathrm{Mn}$ e $\mathrm{Zn}$, por espectrofotometria de absorção atômica; $\mathrm{K}$, por fotometria de chama, e S, por turbidimetria. Os teores de $\mathrm{N}$ foram determinados pelo método semimicro Kjeldahl. No solo, procedeu-se a análise química de rotina, para determinação de $\mathrm{pH}$ em água, $\mathrm{P}, \mathrm{K}, \mathrm{Ca}, \mathrm{Mg}, \mathrm{Al}, \mathrm{H}+\mathrm{Al}$ e matéria orgânica; assim como a soma de bases (SB), saturação por $\mathrm{Al}(\mathrm{m}), \mathrm{CTC}$ efetiva (t), CTC potencial (T) e saturação por bases (V) e a soma dos teores de $\mathrm{N}$-total, $\mathrm{N}-\mathrm{NO}_{3}^{-}$e $\mathrm{N}-\mathrm{NH}_{4}^{+}$. Foi calculado o teor de $\mathrm{N}$ mineral total $\left(\mathrm{N}-\mathrm{NO}_{3}^{-}+\mathrm{N}-\mathrm{NH}_{4}^{+}\right)$, e a relação $\mathrm{N}-\mathrm{NO}_{3}^{-} / \mathrm{N}-\mathrm{NH}_{4}^{+}$. As determinações químicas foram efetuadas conforme Vettori (1969), com modificações pela Embrapa (1979); pH em água (na proporção de 1:2,5 para solo: água); $\mathrm{Ca}^{+2}, \mathrm{Mg}^{+2}$ e $\mathrm{Al}^{+3}$ (extraídos pelo $\mathrm{KCl} 1 \mathrm{~N}$ ); $\mathrm{P}$ e K (extraídos pelo $\mathrm{HCl} 0,05+\mathrm{H}_{2} \mathrm{SO}_{4} 0,025 \mathrm{~N}$ ), e acidez extraível (H+Al) (SMP). O C orgânico foi determinado pelo método colorimétrico (Quaggio \& Raij, 1979) e a porcentagem de matéria orgânica estimada, multiplicando-se o teor de C orgânico por 1,724. Os teores de $\mathrm{N}$-total foram determinados pelo método de micro Kjeldahl, e os teores de $\mathrm{N}-\mathrm{NH}_{4}^{+}$e $\mathrm{N}-\mathrm{NO}_{3}^{-}$por extração com $\mathrm{KCl} 1 \mathrm{~N}$, e destilação com $\mathrm{MgO}$ e liga de Devarda (Bremner, 1965).

Os dados obtidos para produção de matéria seca, concentração e conteúdo de nutrientes nas plantas não passaram por análise estatística, sendo trabalhados como dados auxiliares à discussão dos resultados provenientes da análise de solo. Em cada época de amostragem de solo foi realizada uma análise estatística em separado, todas com o mesmo procedimento. Os fatores manejo da biomassa e cultura foram colocados respectivamente nas parcelas e subparcelas, e o fator profundidade de amostragem de solo foi analisado em faixas. Após as análises de variância, procedeu-se aos desdobramentos das interações. Por não se constituir em objetivo do trabalho avaliar a influência da profundidade de amostragem de solo em cada variável, mas sim, dos fatores manejo e cultura sobre as variáveis em cada uma das profundidades de amostragem de solo, não foram desdobradas as interações triplas nas quais esteve presente o efeito da profundidade, nem realizados testes de comparação de médias no tocante às opções profundidade dentro do manejo e profundidade dentro da cultura. 


\section{RESULTADOS E DISCUSSÃO}

\section{Planta}

Pela análise da Tabela 1, pode-se observar que a maior produção de matéria seca da parte aérea foi obtida com o guandu, $100 \%$ mais que com a crotalária. Calegari (1990), citado por Calegari et al. (1993), testando 19 espécies diferentes de adubos verdes de verão, no sudoeste do Paraná, avaliou a massa seca após o corte em pleno florescimento, e o guandu foi a espécie de maior produção $(13,8 \mathrm{t} / \mathrm{ha})$, seguido pelo milheto $(9,9 \mathrm{t} / \mathrm{ha})$ e pela crotalária-júncea $(9,9$ t/ha).

A maior contribuição do guandu no fornecimento de nutrientes se deve à maior produção de massa seca proporcionando, assim, maiores conteúdos de nutrientes (Tabela 1). Tais resultados obtidos do guandu estão dentro das faixas encontradas por Dolmat (1980), que comparou a quantidade total de nutrientes retornados ao solo por gramíneas e leguminosas e concluiu que a cobertura com leguminosas em pleno vigor pode retornar ao solo de 226 a $353 \mathrm{~kg} / \mathrm{ha}$ de N, 18 a $27 \mathrm{~kg} / \mathrm{ha} \mathrm{de} \mathrm{P}, 85$ a $131 \mathrm{~kg} / \mathrm{ha}$ de K e 15 a $27 \mathrm{~kg} / \mathrm{ha}$ de $\mathrm{Mg}$. Por ser, o sistema radicular do guandu, bem profundo e com boa habilidade de exploração do solo, essa leguminosa apresenta um grande potencial de reciclagem de nutrientes.

De maneira geral, por sua maior contribuição em nutrientes que podem ser devolvidos ao solo após seu manejo, as leguminosas demonstraram maior capacidade de reciclagem de nutrientes em relação à pastagem de $B$. decumbens, que por sua vez mostrou-se bastante debilitada.

É importante salientar que condições espaciais e temporais podem favorecer não só determinadas es- pécies em relação a outras, mas também promover variações de produção dentro da mesma espécie. Fatores como a época de semeadura e o ambiente (luminosidade, temperatura, entre outros) interferem tanto na produção vegetativa quanto na concentração de nutrientes das leguminosas. Miyasaka et al. (1966) verificaram grande variabilidade nos rendimentos de biomassa verde pela crotalária-júncea e guandu em relação ao ambiente, por meio de experimentos conduzidos em vários municípios de São Paulo. Trabalhos mais recentes também têm demonstrado haver interação entre o genótipo e o ambiente afetando a produção de massa seca dos adubos verdes, podendo levar a diferentes observações (Muller et al., 1992; Hundal \& Dhillon, 1993; Böhringer et al., 1994).

O plantio das duas leguminosas em épocas diferentes, no presente trabalho, objetivando propiciar iguais condições de mineralização dos resíduos de ambas, consiste em um fator de interferência sobre a produção de massa seca e a concentração de nutrientes, já que a crotalária-júncea, espécie bastante sensível ao fotoperíodo, teve seu ciclo reduzido, devido ao plantio em fevereiro.

\section{Propriedades químicas do solo}

$\mathrm{Na}$ avaliação química do solo, realizada 90 dias após o manejo das culturas (Tabela 2), observa-se que, independentemente da cultura, nas parcelas onde houve incorporação de biomassa foram encontrados maior teor de $\mathrm{N}-\mathrm{NO}_{3}^{-}+\mathrm{N}-\mathrm{NH}_{4}^{+}$nos primeiros $5 \mathrm{~cm}$ de profundidade, maior teor de $\mathrm{Mg}$ na profundidade de 5 a $10 \mathrm{~cm}$, maior teor de $\mathrm{K}$ nas profundidades de 10 a 20,40 a 60 e 60 a $80 \mathrm{~cm}$, e de $\mathrm{Ca}$ e $\mathrm{Mg}$, e maiores soma de bases, CTC efetiva e saturação por bases nas profundidades de 20 a 40,40 a 60 e

TABELA 1. Produção de matéria seca e fornecimento de nutrientes ao solo pelas culturas, por ocasião do corte (média de três repetições).

\begin{tabular}{|c|c|c|c|c|c|c|c|c|c|c|c|c|}
\hline \multirow[t]{2}{*}{ Cultura } & \multirow{2}{*}{$\begin{array}{c}\text { Matéria } \\
\text { seca }\end{array}$} & \multicolumn{6}{|c|}{ Macronutrientes } & \multicolumn{5}{|c|}{ Micronutrientes } \\
\hline & & $\mathrm{N}$ & $\mathrm{P}$ & $\mathrm{K}$ & $\mathrm{Ca}$ & $\mathrm{Mg}$ & S & $\mathrm{B}$ & $\mathrm{Cu}$ & $\mathrm{Mn}$ & $\mathrm{Zn}$ & $\mathrm{Fe}$ \\
\hline & $(\mathrm{t} / \mathrm{ha})$ & -------- & ------. & ---- ( & a) ---- & ------- & ----- & -------- & ------ & $(\mathrm{g} / \mathrm{ha})$ & ----- & -------- \\
\hline Guandu & 13,2 & 314,6 & 25,9 & 98,4 & 70,3 & 27,7 & 22,0 & 163,0 & 155,1 & 606,4 & 288,7 & $3.889,0$ \\
\hline Crotalária & 6,5 & 136,2 & 9,0 & 30,8 & 60,9 & 22,5 & 12,0 & 98,5 & 58,5 & 340,6 & 150,6 & $1.827,4$ \\
\hline Pastagem & 3,8 & 31,4 & 3,8 & 11,6 & 15,8 & 8,0 & 6,1 & 25,0 & 18,0 & 368,0 & 101,6 & $9.146,3$ \\
\hline
\end{tabular}


60 a $80 \mathrm{~cm}$. Em relação ao Ca na profundidade de 40 a $60 \mathrm{~cm}$, os solos sob as duas formas de manejo (com e sem incorporação) apresentaram teores semelhantes. Nas parcelas onde a biomassa das culturas não foi incorporada, o teor de $\mathrm{K}$ e a saturação por bases foram maiores na profundidade de 0 a $5 \mathrm{~cm}$, assim como a acidez potencial nas profundidades de 20 a 40 e 60 a $80 \mathrm{~cm}$.

Pela análise da Tabela 3, observa-se que em todas as profundidades o teor de $\mathrm{K}$ foi superior no solo sob guandu, seguido pelo solo sob crotalária, destacando-se, nos primeiros $5 \mathrm{~cm}$, o teor aproximadamente $360 \%$ maior desse nutriente no primeiro em comparação com o último, corroborando com a maior contribuição de $\mathrm{K}$ para a matéria seca do guandu (Tabela 1). O guandu é, segundo Amabile (1996), uma espécie com sistema radicular altamente capaz de reciclar e absorver potássio. Vale ressaltar que este nutriente não faz parte de nenhum composto orgânico (Faquin, 1994), e portanto é mais facilmente mineralizável por ocasião da decomposição da matéria orgânica. Além disso, de acordo com Tiwari et al.
(1980), a decomposição da matéria orgânica pode ter efeito solubilizante no K nativo do solo, aumentando sua disponibilidade. Os teores de $\mathrm{Ca}$ e $\mathrm{Mg}$ foram maiores nos solos sob as leguminosas nos primeiros $5 \mathrm{~cm}$, acompanhados, conseqüentemente, por maiores valores de soma de bases, CTC efetiva e saturação por bases. Ainda, quanto ao $\mathrm{Ca}$ e $\mathrm{Mg}$, o solo sob guandu superou os demais nas profundidades entre 10 e $40 \mathrm{~cm}$, com também maiores soma de bases e CTC efetiva. Conforme Testa et al. (1992), o uso de leguminosas capazes de produzir altas quantidades de resíduos permite redução na lixiviação de cátions e aumento na CTC, o qual é acompanhado por aumentos proporcionais nos teores de $\mathrm{Ca}, \mathrm{Mg}$ e $\mathrm{K}$, e conseqüentemente, na soma de bases do solo. Porém, este efeito se dá a longo prazo. No presente trabalho, provavelmente esses maiores valores nos solos sob guandu e crotalária se devem a maior capacidade dessas leguminosas de retornar $\mathrm{Ca}$ e $\mathrm{Mg}$ ao solo através de sua biomassa, em relação à pastagem (Tabela 1). Para a CTC potencial, os solos sob guandu e crotalária se comportaram de maneira se-

TABELA 2. Resultados da análise química do solo em diferentes profundidades, em função das formas de manejo da biomassa, na primeira avaliação (90 dias após o manejo $)^{1}$.

\begin{tabular}{|c|c|c|c|c|c|c|c|c|}
\hline Manejo da biomassa & ------- $\left(\mathrm{mg} / \mathrm{dm}^{3}\right)$------ & $\begin{array}{l}\mathrm{NO}_{3}^{-}+\mathrm{NH}_{4}^{+} \\
\left.\mathrm{ng} / \mathrm{dm}^{3}\right) \text {------ }\end{array}$ & \multicolumn{5}{|c|}{ - } & $\begin{array}{c}\mathrm{V} \\
(\%)\end{array}$ \\
\hline & \multicolumn{8}{|c|}{$0-5 \mathrm{~cm}$} \\
\hline Incorporada & $50,7 \mathrm{~b}$ & $62,8 \mathrm{a}$ & $25,4 a$ & $10,4 \mathrm{a}$ & $51,1 \mathrm{a}$ & $36,9 \mathrm{a}$ & $37,8 \mathrm{a}$ & $40,8 b$ \\
\hline \multirow[t]{2}{*}{ Não incorporada } & $70,1 \mathrm{a}$ & $54,8 \mathrm{~b}$ & $30,3 \mathrm{a}$ & $12,7 \mathrm{a}$ & $43,9 \mathrm{a}$ & $45,4 \mathrm{a}$ & $46,0 \mathrm{a}$ & $49,2 \mathrm{a}$ \\
\hline & \multicolumn{8}{|c|}{$5-10 \mathrm{~cm}$} \\
\hline Incorporada & $31,9 \mathrm{a}$ & $52,8 \mathrm{a}$ & $26,6 \mathrm{a}$ & $11,1 \mathrm{a}$ & $55,0 \mathrm{a}$ & $38,4 a$ & $39,6 \mathrm{a}$ & $39,7 \mathrm{a}$ \\
\hline \multirow[t]{2}{*}{ Não incorporada } & $36,9 \mathrm{a}$ & $54,9 \mathrm{a}$ & $27,6 \mathrm{a}$ & $6,9 b$ & $55,0 \mathrm{a}$ & $39,8 \mathrm{a}$ & $40,6 a$ & $41,2 \mathrm{a}$ \\
\hline & \multicolumn{8}{|c|}{$10-20 \mathrm{~cm}$} \\
\hline Incorporada & $37,9 \mathrm{a}$ & $49,4 \mathrm{a}$ & $20,3 \mathrm{a}$ & $11,3 \mathrm{a}$ & $60,9 \mathrm{a}$ & $30,3 \mathrm{a}$ & $30,9 a$ & $31,9 \mathrm{a}$ \\
\hline \multirow[t]{2}{*}{ Não incorporada } & $21,5 b$ & $46,9 \mathrm{a}$ & $18,6 \mathrm{a}$ & $10,4 \mathrm{a}$ & $67,0 \mathrm{a}$ & $29,3 \mathrm{a}$ & $31,1 \mathrm{a}$ & $30,4 \mathrm{a}$ \\
\hline & \multicolumn{8}{|c|}{$20-40 \mathrm{~cm}$} \\
\hline Incorporada & $23,2 b$ & $49,2 \mathrm{a}$ & $26,0 \mathrm{a}$ & $11,3 \mathrm{a}$ & $50,3 \mathrm{~b}$ & $37,9 \mathrm{a}$ & $39,8 \mathrm{a}$ & $32,5 \mathrm{a}$ \\
\hline \multirow[t]{2}{*}{ Não incorporada } & $46,8 \mathrm{a}$ & $46,4 \mathrm{a}$ & $14,6 b$ & $7,7 \mathrm{~b}$ & $73,9 a$ & $23,3 b$ & $25,7 b$ & $22,9 b$ \\
\hline & \multicolumn{8}{|c|}{$40-60 \mathrm{~cm}$} \\
\hline Incorporada & $37,1 \mathrm{a}$ & $49,3 \mathrm{a}$ & $17,4 \mathrm{a}$ & $8,0 \mathrm{a}$ & $72,3 \mathrm{a}$ & $38,4 \mathrm{a}$ & $39,6 \mathrm{a}$ & $35,7 \mathrm{a}$ \\
\hline \multirow[t]{2}{*}{ Não incorporada } & $21,8 b$ & $48,2 \mathrm{a}$ & $9,7 \mathrm{a}$ & $3,7 b$ & $68,4 \mathrm{a}$ & $14,3 b$ & $15,2 b$ & $11,9 b$ \\
\hline & \multicolumn{8}{|c|}{$60-80 \mathrm{~cm}$} \\
\hline Incorporada & $27,4 \mathrm{a}$ & $46,0 \mathrm{a}$ & $22,5 \mathrm{a}$ & $12,4 \mathrm{a}$ & $60,7 b$ & $44,7 \mathrm{a}$ & $45,5 \mathrm{a}$ & $43,2 \mathrm{a}$ \\
\hline Não incorporada & $14,2 b$ & $45,8 \mathrm{a}$ & $7,9 \mathrm{~b}$ & $4,0 \mathrm{~b}$ & $76,4 a$ & $13,8 \mathrm{~b}$ & $17,0 \mathrm{~b}$ & $12,0 \mathrm{~b}$ \\
\hline
\end{tabular}

${ }^{1}$ Médias seguidas pela mesma letra, para cada variável, em cada profundidade, não diferem entre si (teste $\mathrm{F}, 5 \%$ ). 
melhante em todas as profundidades de amostragem, superando o solo sob pastagem (Tabela 3 ).

As diferenças no tocante aos teores de $\mathrm{N}$ aparecem nos primeiros $10 \mathrm{~cm}$, em que o processo de mineralização da biomassa das leguminosas é mais intenso (Tabela 4). Os adubos verdes se mostraram superiores à pastagem quanto ao teor de $\mathrm{N}$ contido na biomassa, dada a sua capacidade de fixar o $\mathrm{N}$ atmosférico e devido ao seu sistema radicular mais eficiente no aproveitamento dos nutrientes, sendo possível, com sua utilização aumentar os teores de $\mathrm{N}$ do solo, o que tem sido demonstrado na literatura. Em trabalho com adubos verdes de inverno, Heizmann (1985) obteve maiores teores de $\mathrm{N}-\mathrm{NO}_{3}^{-}$ no solo onde esses foram cultivados em sucessão com o milho. Stampford et al. (1994) verificaram um aumento significativo nos teores de $\mathrm{N}-\mathrm{NO}_{3}^{-}$e $\mathrm{N}-\mathrm{NH}_{4}^{+}$do solo após utilização dos adubos verdes labe-labe e mucuna-preta, levando a incrementos de produção na cultura do sorgo em sucessão.

$\mathrm{Na}$ avaliação química do solo realizada 120 dias após o manejo das culturas (Tabela 5), observa-se que a saturação por $\mathrm{Al}$ e os teores de $\mathrm{P}$ foram semelhantes entre os solos sob as três culturas, em praticamente todas as profundidades. As diferenças encontradas quanto ao $\mathrm{P}$ na segunda profundidade, apesar de estatisticamente significativas, são muito pouco expressivas quando se trata de interpretação de resultados, pois os teores de $\mathrm{P}$ foram sempre baixos. Quanto ao $\mathrm{N}-\mathrm{NO}_{3}^{-}$, o solo sob guandu se destacou até os $10 \mathrm{~cm}$, se igualou ao solo sob crotalária na profundidade de 10 a $20 \mathrm{~cm}$, e foi inferior ao último, igualando-se com o solo sob pastagem nas de-

TABELA 3. Resultados da análise química do solo em diferentes profundidades, em função das culturas utilizadas, na primeira avaliação (90 dias após o manejo $)^{1}$.

\begin{tabular}{|c|c|c|c|c|c|c|c|c|}
\hline Cultura & $\begin{array}{c}\mathrm{K} \\
\left(\mathrm{mg} / \mathrm{dm}^{3}\right) \\
\end{array}$ & \multicolumn{6}{|c|}{ - } & $\begin{array}{l}\mathrm{V} \\
(\%)\end{array}$ \\
\hline & \multicolumn{8}{|c|}{$0-5 \mathrm{~cm}$} \\
\hline Guandu & $126 a$ & $36,7 \mathrm{a}$ & $17,0 \mathrm{a}$ & $36,2 b$ & $56,3 \mathrm{a}$ & $56,3 \mathrm{a}$ & $92,5 \mathrm{a}$ & $62 \mathrm{a}$ \\
\hline Crotalária & $35 b$ & $39,5 a$ & $14,2 \mathrm{a}$ & $42,7 b$ & $56,2 \mathrm{a}$ & $56,2 \mathrm{a}$ & $100,7 \mathrm{a}$ & $61 \mathrm{a}$ \\
\hline \multirow[t]{2}{*}{ Pastagem } & $20 \mathrm{~b}$ & $7,4 \mathrm{~b}$ & $3,5 b$ & $63,8 \mathrm{a}$ & $11,0 \mathrm{~b}$ & $13,2 b$ & $83,2 \mathrm{a}$ & $13 b$ \\
\hline & \multicolumn{8}{|c|}{$5-10 \mathrm{~cm}$} \\
\hline Guandu & $60 \mathrm{a}$ & $42,7 \mathrm{a}$ & $16,2 \mathrm{a}$ & $31,8 b$ & $60,3 a$ & $60,3 a$ & $105,8 \mathrm{a}$ & $62 a$ \\
\hline Crotalária & $26 \mathrm{~b}$ & $34,0 \mathrm{~b}$ & $9,3 \mathrm{~b}$ & $61,1 \mathrm{a}$ & $51,0 \mathrm{a}$ & $51,0 \mathrm{a}$ & $101,7 \mathrm{a}$ & $51 \mathrm{~b}$ \\
\hline \multirow[t]{2}{*}{ Pastagem } & $16 \mathrm{~b}$ & $4,5 \mathrm{c}$ & $1,5 \mathrm{c}$ & $72,2 \mathrm{a}$ & $6,0 \mathrm{~b}$ & $9,0 \mathrm{~b}$ & $80,7 \mathrm{~b}$ & $8 \mathrm{c}$ \\
\hline & \multicolumn{8}{|c|}{$10-20 \mathrm{~cm}$} \\
\hline Guandu & $45 \mathrm{a}$ & $35,1 \mathrm{a}$ & $19,2 \mathrm{a}$ & $40,1 b$ & $54,1 \mathrm{a}$ & $54,1 \mathrm{a}$ & $91,8 \mathrm{ab}$ & $57 a$ \\
\hline Crotalária & $28 \mathrm{ab}$ & $16,2 b$ & $11,2 \mathrm{~b}$ & $82,7 \mathrm{a}$ & $25,2 b$ & $26,5 b$ & $107,2 \mathrm{a}$ & $24 b$ \\
\hline \multirow[t]{2}{*}{ Pastagem } & $16 \mathrm{~b}$ & $7,0 \mathrm{c}$ & $2,2 \mathrm{c}$ & $69,2 \mathrm{a}$ & $10,2 \mathrm{c}$ & $12,4 \mathrm{c}$ & $82,8 \mathrm{~b}$ & $13 \mathrm{c}$ \\
\hline & \multicolumn{8}{|c|}{$20-40 \mathrm{~cm}$} \\
\hline Guandu & $68 \mathrm{a}$ & $38,5 \mathrm{a}$ & $18,9 \mathrm{a}$ & $43,2 b$ & $59,4 \mathrm{a}$ & $59,8 \mathrm{a}$ & $94,2 \mathrm{a}$ & $57 \mathrm{a}$ \\
\hline Crotalária & $20 \mathrm{~b}$ & $16,3 b$ & $7,6 \mathrm{~b}$ & $72,3 a$ & $24,4 b$ & $27,6 b$ & $92,5 \mathrm{a}$ & $15 b$ \\
\hline \multirow[t]{2}{*}{ Pastagem } & $18 \mathrm{~b}$ & $6,0 \mathrm{c}$ & $2,0 \mathrm{c}$ & $70,7 \mathrm{a}$ & $8,0 \mathrm{c}$ & $11,0 \mathrm{c}$ & $79,2 \mathrm{a}$ & $11 \mathrm{~b}$ \\
\hline & \multicolumn{8}{|c|}{$40-60 \mathrm{~cm}$} \\
\hline Guandu & $48 \mathrm{a}$ & $13,5 b$ & $9,5 \mathrm{a}$ & $70,8 \mathrm{a}$ & $40,8 \mathrm{a}$ & $42,0 \mathrm{a}$ & $98,5 \mathrm{a}$ & $38 \mathrm{a}$ \\
\hline Crotalária & $30 \mathrm{~b}$ & $21,8 \mathrm{a}$ & $6,2 \mathrm{a}$ & $70,8 \mathrm{a}$ & $31,0 \mathrm{~b}$ & $29,4 b$ & $100,7 \mathrm{a}$ & $24 b$ \\
\hline \multirow[t]{2}{*}{ Pastagem } & $11 \mathrm{c}$ & $5,3 \mathrm{c}$ & $1,8 \mathrm{~b}$ & $69,5 \mathrm{a}$ & $7,3 \mathrm{c}$ & $10,8 \mathrm{c}$ & $76,8 \mathrm{~b}$ & $10 \mathrm{c}$ \\
\hline & \multicolumn{8}{|c|}{$60-80 \mathrm{~cm}$} \\
\hline Guandu & $33 \mathrm{a}$ & $14,4 \mathrm{~b}$ & $11,0 \mathrm{a}$ & $69,2 \mathrm{a}$ & $39,4 \mathrm{a}$ & $41,0 \mathrm{a}$ & $105,6 \mathrm{a}$ & $37 \mathrm{a}$ \\
\hline Crotalária & $19 a b$ & $25,9 a$ & $11,7 \mathrm{a}$ & $69,2 \mathrm{a}$ & $41,0 \mathrm{a}$ & $43,0 \mathrm{a}$ & $101,8 \mathrm{a}$ & $36 \mathrm{a}$ \\
\hline Pastagem & $10 \mathrm{~b}$ & $5,3 \mathrm{c}$ & $2,0 \mathrm{~b}$ & $67,2 \mathrm{a}$ & $7,3 \mathrm{~b}$ & $9,8 \mathrm{~b}$ & $74,5 b$ & $11 \mathrm{~b}$ \\
\hline
\end{tabular}

${ }^{1}$ Médias seguidas pela mesma letra, para cada variável, em cada profundidade, não diferem entre si (Tukey, 5\%). 
mais profundidades. A relação $\mathrm{N}_{-} \mathrm{NO}_{3}^{-} / \mathrm{N}-\mathrm{NH}_{4}^{+}$ mostra tendência semelhante à seguida pelo teor de $\mathrm{N}-\mathrm{NO}_{3}^{-}$. Quanto ao $\mathrm{N}-\mathrm{NO}_{3}^{-}+\mathrm{N}-\mathrm{NH}_{4}^{+}$, os solos sob guandu e crotalária se igualaram até os $20 \mathrm{~cm}$, sendo que abaixo disso o solo sob crotalária apresentou valores mais elevados. Abaixo dos $20 \mathrm{~cm}$, o solo sob crotalária se mostrou superior ao solo sob guandu, devido, provavelmente, à sua maior relação C/N (19,2 contra 16,8 para o guandu), e, conseqüentemente, menor velocidade de decomposição. Segundo Reinrtsen et al. (1984), uma maior relação $\mathrm{C} / \mathrm{N}$ leva à necessidade de um maior período para a completa mineralização pelos microrganismos, com conseqüente demora na liberação dos nutrientes no solo. Assim, a crotalária, por liberar os nutrientes mais lentamente, demonstra ter colaborado mais na fertilidade do solo, nessa época de amostragem, do que o guandu, o qual já teria, provavelmente, passado por sua fase de liberação mais intensa de nutrientes (Tabela 5).

Independentemente da cultura, os teores de P foram semelhantes entre as duas formas de manejo em todas as profundidades, com exceção da segunda, em que o solo que não sofreu incorporação apresentou teores superiores (Tabela 6). Na profundidade de 0 a $5 \mathrm{~cm}$ no solo onde a biomassa não foi incorporada havia duas vezes mais $\mathrm{K}$ do que no solo onde houve incorporação, sendo que abaixo disso os dois solos se igualaram. O teor de $\mathrm{Ca}$, a soma de bases e a CTC efetiva do solo foram superiores no solo que não sofreu incorporação até os $10 \mathrm{~cm}$, e não apresentou mais diferenças abaixo dessa profundidade. A não-incorporação da biomassa, dado o seu menor contato com o solo, pode tornar mais lenta a decom-

TABELA 4. Resultados da análise química do solo em diferentes profundidades, em função das culturas utilizadas, na primeira avaliação (90 dias após o manejo $)^{1}$.

\begin{tabular}{|c|c|c|c|c|}
\hline \multirow[t]{2}{*}{ Cultura } & $\mathrm{N}-\mathrm{NH}_{4}^{+}$ & $\mathrm{N}-\mathrm{NO}_{3}^{-}$ & $\mathrm{NO}_{3}^{-}+\mathrm{NH}_{4}^{+}$ & $\mathrm{NO}_{3}^{-} / \mathrm{NH}_{4}^{+}$ \\
\hline & \multicolumn{4}{|c|}{ - } \\
\hline & \multicolumn{4}{|c|}{$0-5 \mathrm{~cm}$} \\
\hline Guandu & $33 \mathrm{a}$ & $43 \mathrm{a}$ & $76 a$ & $1,23 \mathrm{a}$ \\
\hline Crotalária & $29 \mathrm{a}$ & $27 \mathrm{~b}$ & $57 \mathrm{~b}$ & $0,93 \mathrm{a}$ \\
\hline \multirow[t]{2}{*}{ Pastagem } & $22 b$ & $22 b$ & $44 \mathrm{c}$ & $0,98 \mathrm{a}$ \\
\hline & \multicolumn{4}{|c|}{$5-10 \mathrm{~cm}$} \\
\hline Guandu & $23 \mathrm{a}$ & $42 \mathrm{a}$ & $65 \mathrm{a}$ & $1,85 \mathrm{a}$ \\
\hline Crotalária & $25 \mathrm{a}$ & $26 \mathrm{~b}$ & $51 \mathrm{~b}$ & $1,06 \mathrm{~b}$ \\
\hline \multirow[t]{2}{*}{ Pastagem } & $22 \mathrm{a}$ & $23 b$ & $45 b$ & $1,04 \mathrm{~b}$ \\
\hline & \multicolumn{4}{|c|}{$10-20 \mathrm{~cm}$} \\
\hline Guandu & $22 a$ & $26 a$ & $48 \mathrm{a}$ & $1,22 \mathrm{a}$ \\
\hline Crotalária & $24 a$ & $26 a$ & $50 \mathrm{a}$ & $1,11 \mathrm{a}$ \\
\hline \multirow[t]{2}{*}{ Pastagem } & $24 \mathrm{a}$ & $23 a$ & $46 a$ & $0,97 \mathrm{a}$ \\
\hline & \multicolumn{4}{|c|}{$20-40 \mathrm{~cm}$} \\
\hline Guandu & $25 \mathrm{a}$ & $24 \mathrm{a}$ & $49 \mathrm{a}$ & $0,99 \mathrm{a}$ \\
\hline Crotalaria & $24 \mathrm{a}$ & $24 a$ & $48 \mathrm{a}$ & $1,02 \mathrm{a}$ \\
\hline \multirow[t]{2}{*}{ Pastagem } & $23 a$ & $23 a$ & $46 a$ & $0,99 a$ \\
\hline & \multicolumn{4}{|c|}{$40-60 \mathrm{~cm}$} \\
\hline Guandu & $24 a$ & $26 a$ & $49 a$ & $1,16 \mathrm{a}$ \\
\hline Crotalária & $24 a$ & $26 a$ & $50 \mathrm{a}$ & $1,12 \mathrm{a}$ \\
\hline \multirow[t]{2}{*}{ Pastagem } & $23 a$ & $23 a$ & $47 a$ & $0,96 a$ \\
\hline & \multicolumn{4}{|c|}{$60-80 \mathrm{~cm}$} \\
\hline Guandu & $24 \mathrm{a}$ & $24 a$ & $47 a$ & $1,00 \mathrm{a}$ \\
\hline Crotalária & $22 a$ & $26 a$ & $48 \mathrm{a}$ & $1,20 \mathrm{a}$ \\
\hline Pastagem & $22 a$ & $21 \mathrm{a}$ & $43 a$ & $1,00 \mathrm{a}$ \\
\hline
\end{tabular}

${ }^{1}$ Médias seguidas pela mesma letra, para cada variável, em cada profundidade, não diferem entre si (Tukey, 5\%). 
posição e conduzir a efeitos no solo num maior prazo do que quando se faz a incorporação. Em trabalho de De-Polli \& Chada (1989), verificou-se que quando a biomassa dos adubos verdes foi apenas deixada sobre a superfície do solo, sem que houvesse incorporação, o menor contato com o solo levou a uma decomposição mais lenta.

$\mathrm{Na}$ avaliação química do solo, realizada 150 dias após o manejo das culturas (Tabela 7), observa-se que diferenças entre os tratamentos foram verificadas somente até a profundidade de $20 \mathrm{~cm}$, exceto no $\mathrm{N}-\mathrm{NO}_{3}^{-}$, que na profundidade de 40 a $60 \mathrm{~cm}$ foi maior que no solo sob crotalária. Quanto ao $\mathrm{K}$, apenas na primeira profundidade, houve diferença entre os solos sob as várias culturas, tendo sido os maiores teores encontrados nos solos sob guandu e pastagem. $\mathrm{O}$ teor de $\mathrm{Ca}$ variou nas três primeiras profundi- dades, sendo superior no solo sob pastagem até os $10 \mathrm{~cm}$ superficiais e no solo sob crotalária na profundidade de 10 a $20 \mathrm{~cm}$. O teor de $\mathrm{Mg}$ foi maior no solo sob pastagem na primeira profundidade; abaixo disso, os solos praticamente se igualaram. A relação $\mathrm{N}-\mathrm{NO}_{3}^{-} / \mathrm{N}-\mathrm{NH}_{4}^{+}$foi semelhante nos solos sob as três culturas em todas as profundidades, o que também ocorreu com a acidez potencial, exceto na terceira profundidade, na qual o solo sob guandu demonstrou o maior valor. Soma de bases, CTC efetiva e saturação por bases apresentaram maiores valores no solo sob pastagem até $10 \mathrm{~cm}$ e de 10 a 20 , valores semelhantes para os solos sob pastagem e crotalária. Verifica-se que o solo sob pastagem apresentou tendência de se igualar aos solos sob as leguminosas, superando-os em alguns casos. Esse fato possivelmente se deve à coincidência dessa época de

TABELA 5. Resultados da análise química do solo em diferentes profundidades, em função das culturas utilizadas, na segunda avaliação (120 dias após o manejo) ${ }^{1}$.

\begin{tabular}{|c|c|c|c|c|c|}
\hline Cultura & $\begin{array}{l}\mathrm{m} \\
(\%)\end{array}$ & ----. & $\begin{array}{r}\mathrm{N}-\mathrm{NO}_{3}^{-} \\
\left(\mathrm{mg} / \mathrm{dm}^{3}\right)\end{array}$ & $\mathrm{NO}_{3}^{-}+\mathrm{NH}_{4}^{+}$ & $\mathrm{NO}_{3}^{-} / \mathrm{NH}_{4}^{+}$ \\
\hline & & & $0-5 \mathrm{~cm}$ & & \\
\hline Guandu & $0 \mathrm{a}$ & $3 a$ & $56 a$ & $78 \mathrm{a}$ & $2,58 \mathrm{a}$ \\
\hline Crotalária & $0 \mathrm{a}$ & $4 a$ & $48 b$ & $74 a$ & $1,89 \mathrm{~b}$ \\
\hline \multirow[t]{2}{*}{ Pastagem } & $0 \mathrm{a}$ & $3 a$ & $24 c$ & $44 \mathrm{~b}$ & $1,12 \mathrm{c}$ \\
\hline & & & $5-10 \mathrm{~cm}$ & & \\
\hline Guandu & $0 \mathrm{a}$ & $4 b$ & $50 \mathrm{a}$ & $73 a$ & $2,23 \mathrm{a}$ \\
\hline Crotalária & $0 \mathrm{a}$ & $6 a$ & $43 b$ & $68 \mathrm{a}$ & $1,75 b$ \\
\hline \multirow[t]{2}{*}{ Pastagem } & $0 \mathrm{a}$ & $3 b$ & $21 \mathrm{c}$ & $42 \mathrm{~b}$ & $1,02 \mathrm{c}$ \\
\hline & & & $10-20 \mathrm{~cm}$ & & \\
\hline Guandu & $9 \mathrm{a}$ & $2 a$ & $32 \mathrm{a}$ & $56 a$ & $1,33 \mathrm{a}$ \\
\hline Crotalária & $4 a$ & $2 \mathrm{a}$ & $33 a$ & $60 \mathrm{a}$ & $1,22 \mathrm{ab}$ \\
\hline \multirow[t]{2}{*}{ Pastagem } & $8 \mathrm{a}$ & $2 \mathrm{a}$ & $21 \mathrm{~b}$ & $44 \mathrm{~b}$ & $0,93 b$ \\
\hline & & & $20-40 \mathrm{~cm}$ & & \\
\hline Guandu & $27 \mathrm{a}$ & $1 \mathrm{a}$ & $23 b$ & $46 \mathrm{~b}$ & $1,03 \mathrm{ab}$ \\
\hline Crotalária & $9 b$ & $2 \mathrm{a}$ & $30 \mathrm{a}$ & $57 \mathrm{a}$ & $1,20 \mathrm{a}$ \\
\hline \multirow[t]{2}{*}{ Pastagem } & $24 \mathrm{a}$ & $2 \mathrm{a}$ & $19 b$ & $43 b$ & $0,79 b$ \\
\hline & & & $40-60 \mathrm{~cm}$ & & \\
\hline Guandu & $26 a$ & $1 \mathrm{a}$ & $20 b$ & $41 b$ & $0,98 \mathrm{~b}$ \\
\hline Crotalária & $22 a$ & $1 \mathrm{a}$ & $33 a$ & $54 \mathrm{a}$ & $1,59 \mathrm{a}$ \\
\hline \multirow[t]{2}{*}{ Pastagem } & $31 \mathrm{a}$ & $1 \mathrm{a}$ & $19 b$ & $40 \mathrm{~b}$ & $0,88 \mathrm{~b}$ \\
\hline & & & $60-80 \mathrm{~cm}$ & & \\
\hline Guandu & $27 a b$ & $1 \mathrm{a}$ & $19 b$ & $39 b$ & $0,99 b$ \\
\hline Crotalária & $22 \mathrm{~b}$ & $1 \mathrm{a}$ & $30 \mathrm{a}$ & $50 \mathrm{a}$ & $1,53 \mathrm{a}$ \\
\hline Pastagem & $37 a$ & $1 \mathrm{a}$ & $18 \mathrm{~b}$ & $40 \mathrm{~b}$ & $0,80 \mathrm{~b}$ \\
\hline
\end{tabular}

${ }^{1}$ Médias seguidas pela mesma letra, para cada variável, em cada profundidade, não diferem entre si (Tukey, $5 \%$ ). 
amostragem com a morte de raízes da pastagem de braquiária, que ao se mineralizarem levariam a uma reciclagem de nutrientes no solo. Weaver \& Zink (1946), citados por Alvarenga (1993), afirmam que com o plantio de gramíneas perenes ocorre um aumento anual de material de raiz.

Outro fator que pode ter levado às semelhanças no desempenho das culturas é o fim dos efeitos provocados pela decomposição do material orgânico que havia sido adicionado. Nesta época de amostragem, cinco meses após o corte das culturas, a matéria orgânica proveniente dos adubos verdes já teria sido totalmente decomposta. Ribeiro (1996), estudando os efeitos da incubação do solo com os adubos verdes mucuna-preta e puerária sobre a produção do arroz, atribuiu ao final dos efeitos benéfi- cos provocados pelas leguminosas, o fato de que 180 dias após a incubação não houve diferença nas produções de matéria seca dessa cultura entre os tratamentos com as leguminosas e a testemunha (sem incubação).

Embora tendo-se encontrado expressiva melhoria em algumas propriedades químicas do solo pelo uso dos adubos verdes, os resultados obtidos no presente trabalho indicam que para aproveitar o $\mathrm{N}$ liberado com a decomposição dos adubos verdes, e os outros benefícios que estes podem trazer para a fertilidade do solo, deve-se atentar para sua época de plantio, em intervalos de tempo entre as culturas de interesse econômico, de modo que as melhorias trazidas por sua decomposição no solo possam ser aproveitadas pela cultura sucessora.

TABELA 6. Resultados da análise química do solo em diferentes profundidades, em função das formas de manejo da biomassa, na segunda avaliação (120 dias após o manejo) ${ }^{1}$.

\begin{tabular}{|c|c|c|c|c|c|}
\hline \multirow[t]{2}{*}{ Manejo da biomassa } & $\mathrm{P}$ & $\mathrm{K}$ & $\mathrm{Ca}$ & SB & $\mathrm{t}$ \\
\hline & \multicolumn{2}{|c|}{------- $\left(\mathrm{mg} / \mathrm{dm}^{3}\right)$-------- } & \multicolumn{3}{|c|}{ - $\left(\mathrm{mmol}_{\mathrm{c}} / \mathrm{dm}^{3}\right)$ - } \\
\hline & & & $0-5 \mathrm{~cm}$ & & \\
\hline Incorporada & $3 a$ & $63 b$ & $29,8 b$ & $48,6 b$ & $48,6 b$ \\
\hline \multirow[t]{2}{*}{ Não incorporada } & $4 \mathrm{a}$ & $128 \mathrm{a}$ & $38,4 \mathrm{a}$ & $61,0 \mathrm{a}$ & $61,0 \mathrm{a}$ \\
\hline & & & $5-10 \mathrm{~cm}$ & & \\
\hline Incorporada & $3 b$ & $37 a$ & $27,3 b$ & $44,7 b$ & $44,7 \mathrm{~b}$ \\
\hline \multirow[t]{2}{*}{ Não incorporada } & $5 \mathrm{a}$ & $48 \mathrm{a}$ & $36,7 \mathrm{a}$ & $56,4 \mathrm{a}$ & $56,4 \mathrm{a}$ \\
\hline & & & $10-20 \mathrm{~cm}$ & & \\
\hline Incorporada & $2 \mathrm{a}$ & $27 \mathrm{a}$ & $17,6 \mathrm{a}$ & $29,3 \mathrm{a}$ & $31,0 \mathrm{a}$ \\
\hline \multirow[t]{2}{*}{ Não incorporada } & $2 \mathrm{a}$ & $19 \mathrm{a}$ & $20,3 \mathrm{a}$ & $33,3 \mathrm{a}$ & $34,9 \mathrm{a}$ \\
\hline & & & $20-40 \mathrm{~cm}$ & & \\
\hline Incorporada & $2 \mathrm{a}$ & $21 \mathrm{a}$ & $10,2 \mathrm{a}$ & $15,9 \mathrm{a}$ & $19,2 \mathrm{a}$ \\
\hline \multirow[t]{2}{*}{ Não incorporada } & $1 \mathrm{a}$ & $16 \mathrm{a}$ & $9,8 \mathrm{a}$ & $16,4 \mathrm{a}$ & $19,3 \mathrm{a}$ \\
\hline & & & $40-60 \mathrm{~cm}$ & & \\
\hline Incorporada & 1a & $12 \mathrm{a}$ & $5,8 \mathrm{a}$ & $8,4 a$ & $11,2 \mathrm{a}$ \\
\hline \multirow[t]{2}{*}{ Não incorporada } & $1 \mathrm{a}$ & $12 \mathrm{a}$ & $6,4 a$ & $9,1 \mathrm{a}$ & $12,4 \mathrm{a}$ \\
\hline & & & $60-80 \mathrm{~cm}$ & & \\
\hline Incorporada & 1a & $8 \mathrm{a}$ & $4,2 \mathrm{a}$ & $5,8 \mathrm{a}$ & $8,2 \mathrm{a}$ \\
\hline Não incorporada & $1 \mathrm{a}$ & $9 a$ & $5,4 \mathrm{a}$ & $7,7 \mathrm{a}$ & $10,2 \mathrm{a}$ \\
\hline
\end{tabular}

${ }^{1}$ Médias seguidas pela mesma letra, para cada variável, em cada profundidade, não diferem entre si (teste $\mathrm{F}, 5 \%$ ). 
TABELA 7. Resultados da análise química do solo em diferentes profundidades, em função das culturas utilizadas, na terceira avaliação (150 dias após o manejo) ${ }^{1}$.

\begin{tabular}{|c|c|c|c|c|c|c|c|c|c|}
\hline Cultura & $\begin{array}{c}\mathrm{K} \\
\left(\mathrm{mg} / \mathrm{dm}^{3}\right)\end{array}$ & $\mathrm{Ca}$ & $\mathrm{Mg}$ & $\begin{array}{c}\mathrm{H}+\mathrm{Al} \\
\mathrm{nmol}_{\mathrm{c}} / \mathrm{dm}^{3}\end{array}$ & --------.--- & ----------- & $\begin{array}{l}\mathrm{V} \\
(\%)\end{array}$ & $\begin{array}{c}\mathrm{NO}_{3}^{-} \\
\left(\mathrm{mg} / \mathrm{dm}^{3}\right.\end{array}$ & $\mathrm{NO}_{3}^{-} / \mathrm{NH}_{4}^{+}$ \\
\hline Guandu & $66 a$ & $31,8 b$ & $13,5 b$ & $25,9 \mathrm{a}$ & $\begin{array}{l}0-5 \mathrm{~cm} \\
49,7 \mathrm{~b}\end{array}$ & $49,7 b$ & $68 \mathrm{ab}$ & $27 \mathrm{a}$ & $0,79 \mathrm{a}$ \\
\hline Crotalária & $50 \mathrm{~b}$ & $33,3 b$ & $16,0 \mathrm{~b}$ & $33,8 \mathrm{a}$ & $50,8 b$ & $50,8 b$ & $60 \mathrm{~b}$ & $25 \mathrm{a}$ & $0,84 a$ \\
\hline \multirow[t]{2}{*}{ Pastagem } & $67 \mathrm{a}$ & $42,3 a$ & $21,2 \mathrm{a}$ & $22,5 \mathrm{a}$ & $65,2 \mathrm{a}$ & $65,2 \mathrm{a}$ & $74 a$ & $26 \mathrm{a}$ & $1,08 \mathrm{a}$ \\
\hline & & & & & $5-10 \mathrm{~cm}$ & & & & \\
\hline Guandu & $49 a$ & $32,7 b$ & $13,0 \mathrm{a}$ & $32,3 \mathrm{a}$ & $47,0 \mathrm{~b}$ & $47,0 \mathrm{~b}$ & $60 \mathrm{~b}$ & $23 a$ & $0,76 \mathrm{a}$ \\
\hline Crotalária & $53 \mathrm{a}$ & $35,7 b$ & $16,2 \mathrm{a}$ & $29,5 \mathrm{a}$ & $53,2 \mathrm{ab}$ & $53,2 \mathrm{ab}$ & $64 b$ & $28 \mathrm{a}$ & $0,90 \mathrm{a}$ \\
\hline \multirow[t]{2}{*}{ Pastagem } & $47 a$ & $45,7 \mathrm{a}$ & $16,3 \mathrm{a}$ & $21,5 \mathrm{a}$ & $63,3 \mathrm{a}$ & $63,3 \mathrm{a}$ & $74 a$ & $24 \mathrm{a}$ & $0,89 a$ \\
\hline & & & & & $10-20 \mathrm{~cm}$ & & & & \\
\hline Guandu & $24 \mathrm{a}$ & $15,5 b$ & $9,3 b$ & $62,7 \mathrm{a}$ & $25,7 b$ & $27,2 b$ & $28 \mathrm{~b}$ & $23 a$ & $0,86 \mathrm{a}$ \\
\hline Crotalária & $18 \mathrm{a}$ & $26,3 a$ & $11,0 \mathrm{ab}$ & $47,3 \mathrm{~b}$ & $39,2 \mathrm{a}$ & $40,0 \mathrm{a}$ & $45 \mathrm{a}$ & $24 a$ & $0,81 \mathrm{a}$ \\
\hline \multirow[t]{2}{*}{ Pastagem } & $20 \mathrm{a}$ & $24,0 \mathrm{ab}$ & $13,5 \mathrm{a}$ & $42,8 b$ & $38,0 \mathrm{a}$ & $38,3 \mathrm{a}$ & $47 a$ & $25 \mathrm{a}$ & $0,98 \mathrm{a}$ \\
\hline & & & & & $20-40 \mathrm{~cm}$ & & & & \\
\hline Guandu & $15 \mathrm{a}$ & $6,8 \mathrm{a}$ & $2,7 \mathrm{a}$ & $60,8 \mathrm{a}$ & $9,5 \mathrm{a}$ & $12,0 \mathrm{a}$ & $14 \mathrm{a}$ & $27 \mathrm{a}$ & $0,98 \mathrm{a}$ \\
\hline Crotalária & $13 a$ & $8,3 \mathrm{a}$ & $3,3 a$ & $61,8 \mathrm{a}$ & $11,8 \mathrm{a}$ & $14,8 \mathrm{a}$ & $16 a$ & $28 \mathrm{a}$ & $1,03 \mathrm{a}$ \\
\hline \multirow[t]{2}{*}{ Pastagem } & $16 \mathrm{a}$ & $8,9 a$ & $2,8 \mathrm{a}$ & $58,2 \mathrm{a}$ & $18,2 \mathrm{a}$ & $20,2 \mathrm{a}$ & $24 \mathrm{a}$ & $23 \mathrm{a}$ & $0,95 \mathrm{a}$ \\
\hline & & & & & $40-60 \mathrm{~cm}$ & & & & \\
\hline Guandu & $9 a$ & $5,5 \mathrm{a}$ & $2,0 \mathrm{a}$ & $57,7 \mathrm{a}$ & $7,5 \mathrm{a}$ & $9,7 \mathrm{a}$ & $12 \mathrm{a}$ & $23 b$ & $0,86 \mathrm{a}$ \\
\hline Crotalária & $12 \mathrm{a}$ & $6,3 a$ & $1,8 \mathrm{a}$ & $61,5 \mathrm{a}$ & $8,2 \mathrm{a}$ & $11,5 \mathrm{a}$ & $12 \mathrm{a}$ & $35 a$ & $1,20 \mathrm{a}$ \\
\hline \multirow[t]{2}{*}{ Pastagem } & $10 \mathrm{a}$ & $5,5 \mathrm{a}$ & $2,5 \mathrm{a}$ & $51,2 \mathrm{a}$ & $8,0 \mathrm{a}$ & $10,6 a$ & $13 a$ & $25 b$ & $0,98 \mathrm{a}$ \\
\hline & & & & & $60-80 \mathrm{~cm}$ & & & & \\
\hline Guandu & $8 \mathrm{a}$ & $5,3 \mathrm{a}$ & $2,0 \mathrm{a}$ & $56,2 \mathrm{a}$ & $7,3 \mathrm{a}$ & $9,3 \mathrm{a}$ & $12 \mathrm{a}$ & $23 a$ & $0,90 \mathrm{a}$ \\
\hline Crotalária & $12 \mathrm{a}$ & $5,2 \mathrm{a}$ & $1,8 \mathrm{a}$ & $58,5 \mathrm{a}$ & $7,0 \mathrm{a}$ & $9,8 \mathrm{a}$ & $13 a$ & $32 \mathrm{a}$ & $1,25 \mathrm{a}$ \\
\hline Pastagem & $8 \mathrm{a}$ & $6,0 \mathrm{a}$ & $2,0 \mathrm{a}$ & $48,8 \mathrm{a}$ & $8,0 \mathrm{a}$ & $9,7 \mathrm{a}$ & $11 \mathrm{a}$ & $24 \mathrm{a}$ & $1,01 \mathrm{a}$ \\
\hline
\end{tabular}

${ }^{1}$ Médias seguidas pela mesma letra, para cada variável, em cada profundidade, não diferem entre si (Tukey, $5 \%$ ).

\section{CONCLUSÕES}

1. As leguminosas utilizadas como adubo verde apresentam maior capacidade de reciclagem e mobilização de nutrientes, em comparação com a pastagem de braquiária, graças a suas maiores concentrações de nutrientes na biomassa.

2. A contribuição do guandu nas propriedades químicas do solo se dá num menor espaço de tempo que a da crotalária-júncea.

3. Aos 150 dias após o manejo, não se observa nenhum efeito dos adubos verdes sobre a fertilidade do solo, o que evidencia o fim dos efeitos benéficos provenientes de seus processos de decomposição e mineralização.

4. A incorporação da biomassa acelera sua decomposição e favorece os efeitos benéficos dos adubos verdes.

\section{REFERÊNCIAS}

ALVARENGA, R.C. Potencialidades de adubos verdes para conservação e recuperação de solos. Viçosa : UFV, 1993. 112p. Tese de Doutorado.

AMABILE, R.F. Comportamento de adubos verdes em épocas de semeadura nos cerrados do Brasil central. Piracicaba : ESALQ, 1996. 123p. Dissertação de Mestrado.

ANDRADE, L.A. de B.; ABRAHÃO, J.T.M.; GODOY, O.P. Efeitos da incorporação de Crotalaria juncea L. sobre a cana-de-açúcar. I. Efeito no desenvolvimento inicial. STAB, Piracicaba, v.2, n.3, p.40-43, 1984.

BÖHRINGER, A.; TAMÓ, M.; DREYER, H.M. Growth and productivity of pigeonpea (Cajanus cajan) genotypes for use in alley cropping and their 
interactions with the environment. Experimental Agriculture, v.30, p.207-215, 1994.

BREMNER, J.M. Total nitrogen. In: BLACK, C.A.; EVANS, D.D.; WHITE, J.L.; ENSMINGER, F.E.; CLARK, F.E. (Eds.). Methods of soil analysis on chemical and microbiological properties. Wisconsin : American Society of Agronomy, 1965. v.9, Part. 2, p.1149-1178

CACERES, N.T. Adubação verde com leguminosas em rotação com cana-de-açúcar (Saccharum spp.). Piracicaba : ESALQ, 1994. 45p. Dissertação de Mestrado.

CALEGARI, A.; MONDARDO, A.; BULISANI, E.A.; WILDNER, L. do P.; COSTA, M.B.B. da; ALCÂNTARA, P.B.; MIYASAKA, S.; AMADO, J.T. Aspectos gerais da adubação verde. In: COSTA, M.B.B. da. (Coord.). Adubação verde no sul do Brasil. 2.ed. Rio de Janeiro : AS-PTA, 1993. 346p.

CAMARGO, A.P. de; FREIRE, E.S.; VENTURINI, W.R. Adubação da batata-doce em São Paulo. Parte II Efeito do calcário e de vários adubos. Bragantia, Campinas, v.21, n.1, p.325-339, 1968.

COMISSÃO DE FERTILIZANTES DO SOLO DO ESTADO DE MINAS GERAIS. Recomendações para o uso de corretivos e fertilizantes em Minas Gerais: 4a aproximação. Lavras, 1989. 176p.

DE-POLLI, H.; CHADA, S. de S. Adubação verde incorporada ou em cobertura na produção de milho em solo de baixo potencial de produtividade. Revista Brasileira de Ciência do Solo, Campinas, v.13, n.3, p.287-293, set./dez. 1989.

DOLMAT, M.T. Role of legumes covers. The effects on growth and yield. In: RUBBER RESEARCH INSTITUTE OF MALAYSIA. RRIM training manual rubber planting and nursery techniques. Kuala Lumpur, 1980. p.149-157.

EMBRAPA. Serviço Nacional de Levantamento e Conservação de Solos (Rio de Janeiro, RJ). Manual de métodos de análise de solo. Rio de Janeiro : Ministério da Agricultura, 1979. n.p.

FAQUIN, V. Nutrição mineral de plantas. Lavras : ESAL/FAEPE, 1994. 227p.

HEIZMANN, F.X. Resíduos culturais de inverno e assimilação de nitrogênio por cultura de verão. Pesquisa Agropecuária Brasileira, Brasília, v.9, n.20, p.1021-1030, set. 1985.
HUNDAL, H.S.; DHILLON, N.S. Influence of green manures on $\mathrm{P}$ use efficiency in rice. International Rice Research Newsletter, v.18, n.1, p.43-44, 1993.

KIEHL, E.J. Contribuição para o estudo da poda e da decomposição de adubos verdes. Piracicaba : ESALQ, 1960. 113p. Tese de Livre Docência.

MALAVOLTA, E.; VITTI, G.C.; OLIVEIRA, S.A. de Avaliação do estado nutricional de plantas, princípios e aplicações. Piracicaba: POTAFOS, 1989. $201 \mathrm{p}$.

MIYASAKA, S.; CAMARGO, O.A. de; CAVALERI, P.A.; GODOY, I.J. de; WERNER, J.C.; CURI, S.M.; LOMBARDI NETO, F.; MEDINA, J.C.; CERVELLINI, G.S.; BULISANI, E.A. Adubação orgânica, adubação verde e rotação de culturas no Estado de São Paulo. In: FUNDAÇÃO CARGILL. Adubação orgânica, adubação verde e rotação de culturas no Estado de São Paulo. Campinas, 1984. Parte 1, p.1-109.

MIYASAKA, S.; FREIRE, E.S.; MASCARENHAS, H.A.A.; NERY, C.; CAMPANA, M.; SORDI, G. de. Efeito da adubação verde com uma gramínea e quatro leguminosas sobre a produção do feijoeiro "da seca", em terra-roxa misturada. Bragantia, Campinas, v.25, n.25, p.277-289, 1966.

MULLER, N.R.M.; MULLER, A.A.; OLIVEIRA, R.P. de; MARADEI, N.R. Uso de leguminosas para produção de biomassa. In: EMBRAPA. Centro de Pesquisa Agropecuária do Trópico Úmido (Belém, PA). Programas nacionais de pesquisa para a Região do Trópico Úmido. Belém, 1992. p.111-120.

QUAGGIO, J.A.; RAIJ, B. van. Comparação de métodos rápidos para a determinação da matéria orgânica em solos. Revista Brasileira de Ciência do Solo, Campinas, v.3, n.3, p.184-187, set./dez. 1979.

REINRTSEN, S.A.; ELIOTT, L.F.; COCHRAN, V.L. Role of available carbon and nitrogen In determining the rate of wheat straw decomposition. Soil Biology and Biochemistry, Oxford, v.16, n.5, p.459-464, 1984.

RIBEIRO, G. de A. Efeito de períodos de incubação de adubos verdes (mucuna e puerária) na liberação de nitrogênio $\left({ }^{15} \mathrm{~N}\right)$ e enxofre $\left({ }^{35} \mathrm{~S}\right)$ para 0 arroz. Piracicaba : ESALQ, 1996. 82p. Dissertação de Mestrado.

STAMPFORD, N.P.; ALBUQUERQUE, M.H.; SANTOS, D.R. Aproveitamento do nitrogênio pelo sorgo 
em sucessão a leguminosas incorporadas em diferentes épocas de corte. Revista Brasileira de Ciência do Solo, Campinas, v.18, n.2, p.221-227, maio/ago.1994.

TESTA, V.M.; TEIXEIRA, L.A.J.; MIELNICZUK, J. Características químicas de um Podzólico Vermelho-Escuro afetadas por sistemas de culturas. Revista Brasileira de Ciência do Solo, Campinas, v.1, n.16, p.107-114, jan./abr. 1992.
TIWARI, K.N.; TIWARI, S.P.; PATHAK, A.N. Studies on green manuring of rice in double cropping system in a partially reclained saline sodic soil. Indian Journal of Agronomy, Palampur, v.25, n.1, p.136-145, Mar. 1980.

VETTORI, L. Métodos de análise de solo. Rio de Janeiro : Ministério da Agricultura. Equipe de Pedologia e Fertilidade do Solo, 1969. 24p. (Boletim técnico, 7). 